\title{
O PARADOXO HIDROSTÁtico DE GALILEU E A LEI DE ARQUIMEDES
}

\author{
Fernando Lang da Silveira \\ Instituto de Física - UFRGS \\ Porto Alegre - RS \\ Alexandre Medeiros \\ Departamento de Física - UFRPE \\ Recife - PE
}

\section{Resumo}

A pedagogia tradicional tem subestimado a presença de certos mistérios e paradoxos no ensino da Física. Abordagens indutivistas ingênuas na educação em ciência têm também contribuído para que alguns importantes pressupostos teóricos sejam usualmente ignorados. Uma análise histórica, conceitual e experimental do desenvolvimento da Física pode contribuir positivamente para restaurar a importância de tais pressupostos no ensino da Física. Neste artigo, analisamos o assim denominado Paradoxo Hidrostático de Galileu, um enigma conectado com a Lei de Arquimedes para a Hidrostática. Uma análise experimental desse tema é apresentada de forma fotograficamente bem documentada e conclusões são tiradas como um exemplo para ilustrar a extensão em que uma competente análise de pressupostos ocultos pode contribuir para subverter a educação tradicional e ortodoxa em Física.

Palavras-chave: Lei de Arquimedes; Paradoxo de Galileu; estática de fluidos.

\footnotetext{
Galilean Hydrostatic Paradox and Archimedes Law

* Recebido: outubro de 2008.

Aceito: março de 2009.
} 


\begin{abstract}
Traditional pedagogy has underestimated the presence of some mysteries and paradoxes in Physics teaching. Nä̈ve inductivistic approaches in Science education have also contributed to the fact that some important theoretical assumptions are usually discarded. Historical, conceptual and experimental analysis of Physics development can positively contribute to restore the importance of such assumptions in physics teaching. In this article we analyze the so called Galilean Hydrostatic Paradox, a mystery directly connected to Archimedes Law of Hydrostatics. Experimental analysis of this theme is presented in a well-documented photographic way and conclusions are drawn as an example to show the extent to which a competent analysis of such hidden assumptions can subvert traditional and orthodox education in Physics.
\end{abstract}

Keywords: Archimedes Law; Galilean Hydrostatic Paradox; Statics of Fluids.

\title{
I. Mistérios e mitos no Ensino da Física
}

O ensino tradicional da Física tem padecido, dentre outros problemas, de uma exagerada tendência indutivista ingênua que, ignorando a complexidade da produção do conhecimento científico, costuma vincular a geração de ideias e teorias às observações empíricas, de uma forma direta e praticamente sem qualquer intermediação teórica (MEDEIROS; BEZERRA FILHO, 2000; MEDEIROS; MEDEIROS, 2001). Esse indutivismo ingênuo e simplificador reengendra a história da ciência, na tentativa inglória de justificar as práticas pedagógicas nele fundamentadas, causando assim equívocos e distorções que precisam ser devidamente reparados (SILVEIRA; OSTERMANN, 2002; MEDEIROS; MEDEIROS, 2002; SILVEIRA; PEDUZZI, 2006). A atitude indutivista ingênua no ensino da Física simplifica a sua história, ao ignorar a importância dos pressupostos teóricos e da existência de contextos de validade nas afirmações científicas, produzindo frequentemente uma série de mitos e enigmas que necessitam ser esclarecidos (MEDEIROS; LIMA JR, 2000; MOURA; CANALLE, 2001; MEDEIROS; MEDEIROS, 2002; SILVEIRA; AXT, 2006; SILVEIRA; MEDEIROS, 2006). Essa dimensão do mistério, entretanto, pode ter também o seu lado positivo, pois o seu 
desvelar no ato educativo, quando acompanhado de uma forte carga motivacional, favorece a aprendizagem dos conteúdos científicos abordados (MEDEIROS; MEDEIROS, 2003; SILVEIRA; AXT, 2004; MEDEIROS; MEDEIROS, 2004; SILVEIRA; AXT, 2005; MEDEIROS, 2005). Dentre tais mitos e mistérios a serem desvendados, boa parte está vinculada à mecânica dos fluidos e tem sido objeto de investigações recentes (MEDEIROS, 1999; MEDEIROS; CARMO, 2003; MEDEIROS; MEDEIROS; CARMO, 2003; SILVEIRA; LEVIN, 2004).

Parte relevante das muitas investigações pedagógicas relativas à mecânica dos fluidos se refere a questões hidrostáticas e à existência de aparentes paradoxos (BUZDIN; KROTOV, 1990; WILSON, 1995; BARTLETT, 1997; BETYAEV, 1998; FIOLHAIS, 1999; FONTANA; DI CÁPUA, 2005). No tocante a tais questões e aos paradoxos hidrostáticos, a Lei de Arquimedes ${ }^{1}$ tem sido um alvo preferencial de vários estudos educacionais, sob diferentes enfoques pedagógicos (HODDESON, 1972; HAYN, 1975; HALFORD; BROWN; THOMPSON, 1986; MAMOLA, 1995; SILVA, 1998; GUIMARÃES, 1999; SCONYERS; TRAUTWEIN, 2000; COOPER, 2001; KAPLAN, 2003; LOVERUDE; KAUTZ; HERON, 2003; HUGHES, 2005). Dentre tais estudos, alguns têm dado especial atenção a certos aspectos da abordagem histórica que pode cercar a Lei de Arquimedes (BARDIS, 1984; SNIR, 1991; MARTINS, 2000). Estudos clássicos sobre a história da mecânica dos fluidos servem igualmente para que possamos perceber a complexidade existente e por vezes ignorada na construção da Lei de Arquimedes (DUGAS, 1988; MACH, 1989; TOKATY, 1994). Em meio a tal complexidade, surge uma questão enigmática e interessante, ligada ao enunciado e ao contexto de validade da Lei de Arquimedes, e que originalmente é atribuída a Galileu. Em seu influente trabalho de 1991, Joseph Snir já alertava com bastante propriedade para a importância pedagógica deste paradoxo histórico, apontando direções explicativas,

Apesar de a maioria dos livros-textos de Física Geral, seja de Ensino Médio ou de Ensino Superior, denominar de Princípio o conhecido enunciado de Arquimedes, optaremos por denominá-lo de Lei de Arquimedes. Um princípio se constitui em uma "proposição posta no início de uma dedução, não sendo deduzida de nenhuma outra no sistema considerado" (LALANDE, 1993; p. 861). Ora, no texto de Arquimedes, Sobre os corpos flutuantes (ASSIS, 1996), é na Proposição 5, demonstrada a partir do Postulado 1 e das proposições que lhe antecedem e já foram provadas, que reconhecemos o famoso enunciado de Arquimedes. Dessa forma, do ponto de vista lógico, aquele enunciado é um teorema - "enunciado demonstrado em uma teoria" (LALANDE, 1993; p. 1126) - e, portanto, não há que chamá-lo de princípio. O termo lei é mais adequado, pois é neutro em relação à ideia de o enunciado ser um princípio ou um teorema. 
sem, contudo, explorá-las em sua devida profundidade, não apresentando imagens que pudessem se constituir em evidências cabais do mesmo. O presente trabalho é uma tentativa de resgatar o referido Paradoxo Hidrostático de Galileu, apresentando imagens convincentes, assim como modelos explicativos que possam subsidiar o trabalho dos professores que desejem lidar com o ensino da hidrostática em uma perspectiva cognitivamente mais desafiadora.

\section{O mito e o mistério da Lei de Arquimedes}

O mistério do Paradoxo Hidrostático de Galileu provém do fato de que comumente não nos apercebemos que a Lei de Arquimedes, da forma como costuma ser apresentada "experimentalmente" nos livros-texto, nos induz fatalmente a um erro de avaliação. A referida lei afirma que: "todo corpo mergulhado em um líquido sofre um empuxo de baixo para cima igual ao peso do fluido por ele deslocado". A principal peça de convencimento educacional comumente oferecida pelos livros didáticos de Física para que o aprendiz aceite a validade de tal afirmativa de Arquimedes é um "experimento" no qual um corpo é inicialmente colocado a flutuar em um vaso já repleto de água. No referido "experimento" - usualmente ilustrado nos livros - toda a água que extravasa do recipiente é recolhida e convenientemente pesada. $\mathrm{O}$ resultado mostra que o peso desta água deslocada é igual ao peso do corpo colocado a flutuar. O tal experimento parece convincente, a julgar pelo uso secular que os autores de livros didáticos de Física têm feito do mesmo, desde que Gravesande (1688-1742) e Musschenbroek (1692-1761) o utilizaram de forma pioneira em seus livros ainda no início do século XVIII. Desde então, tal experimento $^{2}$ tem sido assumido como uma peça de evidência válida, convincente e insofismável da referida Lei de Arquimedes, sem que se leve em conta que a justeza desse argumento já houvesse sido criticada no século XVII por Galileu, dando origem ao seu célebre Paradoxo Hidrostático.

2 É importante notar que no texto de Arquimedes (287 a.C.-212 a.C.), Sobre os corpos flutuantes (ASSIS, 1996), não são apresentados quaisquer fatos ou evidências experimentais. O referido texto pode ser encontrado em: $<$ http://www.ifi.unicamp.br/ assis/Revista-

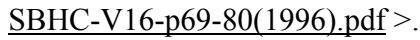




\section{O paradoxo hidrostático de Galileu}

Galileu (1564-1642) idealizou e realizou um experimento paradoxal para o enunciado tradicional da Lei de Arquimedes: todo corpo mergulhado em um líquido sofre um empuxo de baixo para cima igual ao peso do fluido por ele deslocado.

Imaginemos que, em um recipiente, exista um volume de fluido com peso menor do que o peso do corpo flutuante. A Foto 1 da Fig. 1 mostra uma lata de cerveja (cheia de cerveja) com volume aproximado de $350 \mathrm{ml}$ e um recipiente contendo $230 \mathrm{ml}$ de água (colorida com tinta amarela). Quando a lata flutua em água, conforme se observa na Foto 2 da mesma figura, encontra-se quase que completamente imersa; assim sendo, segundo o enunciado usual da Lei de Arquimedes, deveria deslocar quase $350 \mathrm{ml}$ de água. Apesar de não existir no recipiente esse volume, a Foto 2 mostra a lata flutuando. Assim, fica estabelecido um paradoxo em relação ao enunciado usual da Lei de Arquimedes, pois um corpo pode flutuar mesmo quando o volume de fluido é menor do que aquele que o corpo precisaria deslocar. Ou ainda, o Paradoxo Hidrostático de Galileu consiste na afirmação de que um corpo pode flutuar em um fluido mesmo quando o peso de fluido disponível é menor do que o peso do corpo.

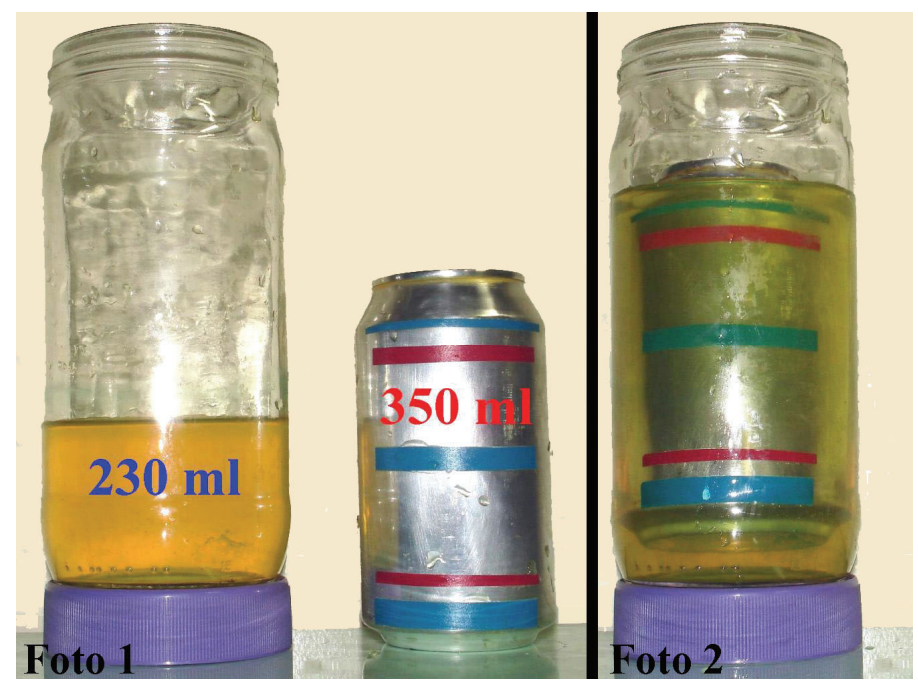

Fig. 1 - Paradoxo de Galileu: corpo flutuando em um recipiente onde não existe o volume de líquido requerido pelo enunciado tradicional da Lei de Arquimedes. 
A desobediência ao enunciado tradicional da Lei de Arquimedes, na situação mostrada na Fig. 1, é uma consequência de que esta proposição é válida somente quando o nível do líquido no recipiente antes e depois da introdução do corpo flutuante permanecer o mesmo. Essa condição é satisfeita se o recipiente estiver inicialmente com a sua capacidade máxima preenchida e, então, transbordar quando da imersão do corpo, ou ainda, quando o volume do corpo for desprezível em relação ao volume de fluido no recipiente. Quando a imersão do corpo no fluido afetar o nível do fluido, mostraremos que o enunciado tradicional da Lei de Arquimedes deixa de ser válido. $\mathrm{O}$ empuxo, nesse caso, é maior do que o peso do volume de fluido efetivamente deslocado.

Nas próximas seções, obteremos a condição de flutuação e resolveremos o Paradoxo de Galileu por intermédio de três abordagens alternativas. Em todas elas utilizaremos uma matemática acessível a alunos de Ensino Médio.

\section{O Paradoxo de Galileu e o modelo da balança hidrostática}

A Fig. 2 representa, de forma idealizada, o sistema real que utilizamos (vide as fotos da Figura 1) para exemplificar o Paradoxo de Galileu. A lata de cerveja e o recipiente são idealizados por dois corpos cilíndricos de altura $H_{i}$ e raio da base $R_{i}\left(i=1\right.$ para a lata de cerveja e $i=2$ para o recipiente), sendo $R_{2}>R_{l}$.
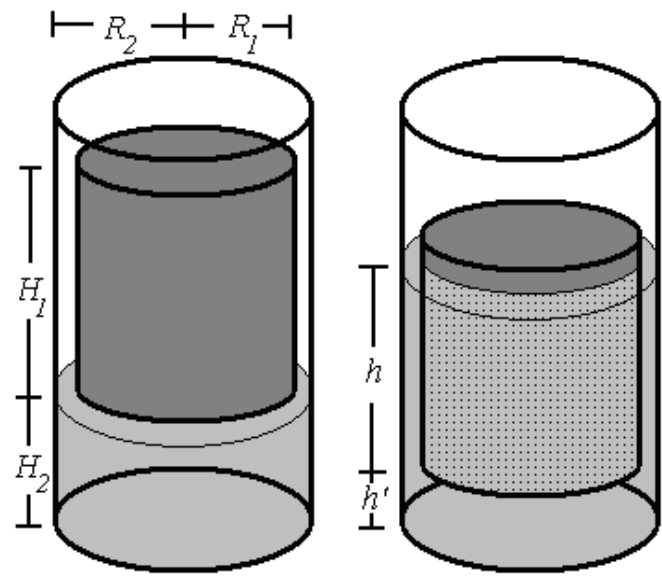

Fig. 2 - Paradoxo de Galileu: o volume de líquido no recipiente é menor do que o volume submerso do corpo flutuante. 
O Paradoxo de Galileu pode ser elucidado modelando o sistema representado na Fig. 2 como uma balança hidrostática ideal (conforme a Fig. 3). A Fig. 3 representa o corpo tocando a superfície do líquido e, portanto, a balança está desequilibrada. O corpo cilíndrico no ramo direito da balança se ajusta perfeitamente a esse vaso e pode deslizar sobre as suas paredes sem atrito, deslocando o líquido de um vaso para outro através de uma conexão no fundo da balança.

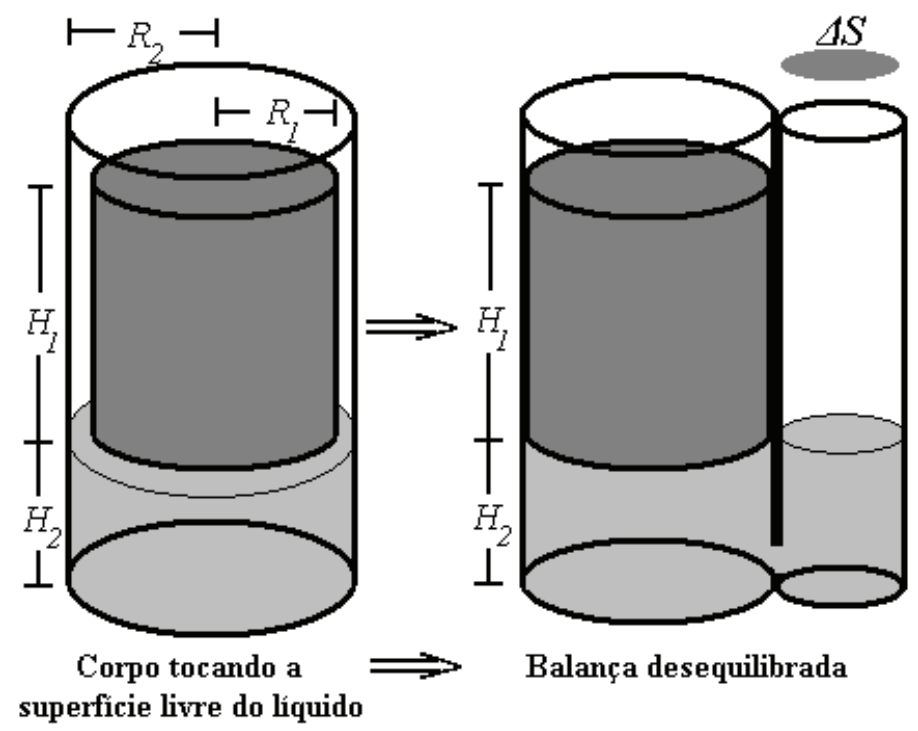

Fig. 3 - Modelo da balança hidrostática para a solução do Paradoxo de Galileu: balança inicialmente desequilibrada.

Para que a balança equivalha ao sistema real idealizado, a seção transversal do ramo direito da balança desequilibrada, na Fig. 3, deve possuir a mesma área $\Delta S$ da seção transversal da região compreendida entre o corpo e a parede lateral do recipiente original. Ou seja, a área $\Delta S$ deve ser igual à área da coroa circular com raio externo $R_{2}$ e raio interno $R_{1}$ mostrada na Fig. 3. Dessa forma,

$$
\Delta S=\pi\left(R_{2}^{2}-R_{1}^{2}\right)
$$

A Fig. 4 representa agora o corpo flutuando em repouso no recipiente da esquerda, equivalendo à balança finalmente equilibrada. Portanto, houve o deslo- 
camento de líquido de um vaso para o outro graças à conexão no fundo dos dois vasos.

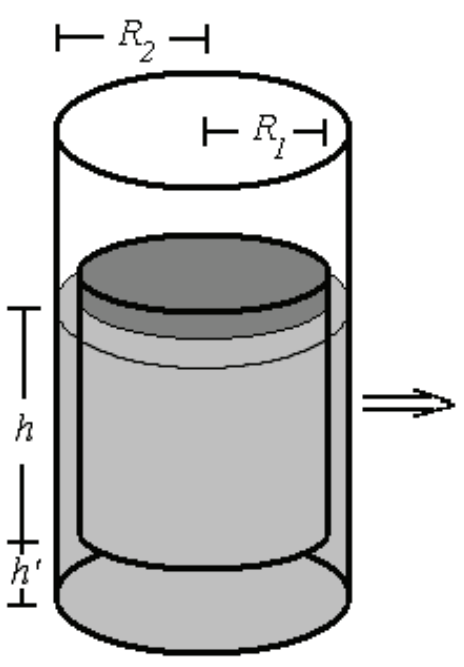

Corpo flutuando

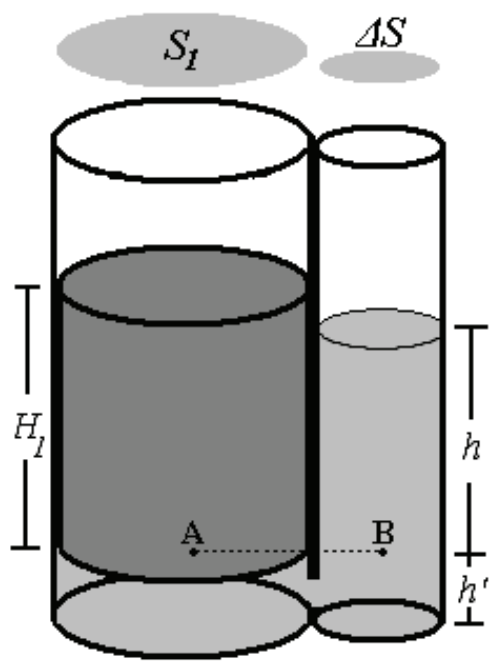

Balança equilibrada

Fig. 4 - Modelo da balança hidrostática para a solução do Paradoxo de Galileu: balança equilibrada.

Quando o sistema se encontra em equilíbrio, pontos no mesmo nível dentro do fluido estão à mesma pressão. Consideremos, então, um ponto $\mathrm{A}$ imediatamente abaixo do corpo flutuante e um ponto $\mathrm{B}$, no mesmo nível, no interior do outro ramo da balança, ambos a uma altura $h$ ' em relação ao fundo da balança (vide a Fig. 4). Acima de A encontramos o corpo que flutua e acima deste a atmosfera. Portanto, para calcularmos a pressão $p_{A} \mathrm{em} \mathrm{A}$, teremos que adicionar, à pressão atmosférica $p_{a t m}$, um incremento de pressão $\Delta p_{\text {Corpo }}$ produzido pelo corpo flutuante. Esse incremento de pressão, quando o corpo está em repouso, é igual ao peso do corpo $P_{\text {Corpo }}$ dividido pela área $S_{I}$ da base do corpo. Além disso, o peso do corpo é igual ao produto da massa $m_{\text {Corpo }}$ do corpo, pela intensidade $g$ do campo gravitacional. Dessa forma,

$$
p_{A}=p_{\text {atm }}+\Delta p_{\text {Corpo }}=p_{\text {atm }}+\frac{P_{\text {Corpo }}}{S_{1}}=p_{\text {atm }}+\frac{m_{\text {Corpo }} g}{S_{1}} .
$$


Acima do ponto B encontramos uma coluna de líquido com altura $h$, e acima desta, a atmosfera. Da mesma forma, a pressão $p_{B}$ em B é calculada por

$$
p_{B}=p_{\text {atm }}+\Delta p_{\text {Coluna }}=p_{\text {atm }}+\frac{P_{\text {Coluna }}}{S_{1}}=p_{\text {atm }}+\frac{m_{\text {Coluna }} g}{S_{1}} .
$$

Para que a balança esteja em equilíbrio, a pressão no ponto A deve ser igual à pressão no ponto $\mathrm{B}$, isto é,

$$
p_{A}=p_{B} .
$$

Substituindo, para $p_{A}$ e $p_{B}$, as expressões obtidas em (2) e em (3), respectivamente, reescrevemos (4) sob a forma:

$$
\frac{m_{\text {Corpo }}}{S_{l}}=\frac{m_{\text {Coluna }}}{\Delta S} .
$$

Se em (5) expressarmos as massas do corpo e da coluna de líquido em função das densidades, $\rho_{\text {Sólido }}$ para o material do qual é feito o corpo sólido e $\rho_{\text {Liquido }}$ para o líquido na coluna, bem como de seus respectivos volumes $V_{l}$ e $V_{2}$, obtemos:

$$
\frac{\rho_{\text {Sólido }} V_{1}}{S_{1}}=\frac{\rho_{\text {Liquido }} V_{2}}{\Delta S} .
$$

Usando o fato de que a razão entre o volume de um cilindro pela área da sua seção transversal é a altura do cilindro, vemos que, de acordo com a Fig. 4:

$$
\rho_{\text {Sólido }} H_{1}=\rho_{\text {Liquido }} h,
$$

ou ainda,

$$
\rho_{\text {Sólido }}=\frac{h}{H_{1}} \rho_{\text {Liquido }} .
$$

Para que o corpo flutue, não pode haver líquido por cima dele e, portanto, conclui-se que $H_{l}$ não pode ser menor que $h$. Assim, apesar da equação (8) verificar-se para a balança, quaisquer que sejam os valores das alturas envolvidas, sua validade para o sistema modelado pela balança fica condicionada à restrição:

$$
\frac{h}{H_{1}} \leq 1
$$

Da equação (8), juntamente com a restrição (9), tem-se que a condição de flutuação é:

$$
\rho_{\text {Sólido }} \leq \rho_{\text {Liquido }} .
$$


O resultado (8) ou (10) mostra que a condição de flutuação não faz referência direta a volumes deslocados, mas sim ao desnível entre a base do corpo flutuante e a superfície de separação do líquido com a atmosfera, ou ainda, à densidade do material que constitui o corpo sólido e à densidade do líquido.

Para que exista a coluna de líquido com altura $h$ - que é o que realmente importa para ocorrer a flutuação! - o corpo efetivamente poderá deslocar muito pouco fluido (ver Equação (1)), se a diferença entre o raio $R_{l}$ do corpo e $R_{2}$ do recipiente for pequena. Havendo o deslocamento de líquido, a altura do nível do fluido no recipiente aumenta; para um dado volume de líquido deslocado, quanto menor for a área $\Delta S$ da seção transversal do ramo direito da balança (ver Figura 4), maior será a altura do nível do fluido no recipiente. A seguir, demonstraremos a relação existente entre o volume $V_{\text {Desl }}$ de líquido efetivamente deslocado e o volume $V_{A r q}$ do líquido referido no enunciado usual da Lei de Arquimedes. Os dois volumes estão representados pelas regiões assinaladas (indicadas por pontos escuros) da Fig. 5.

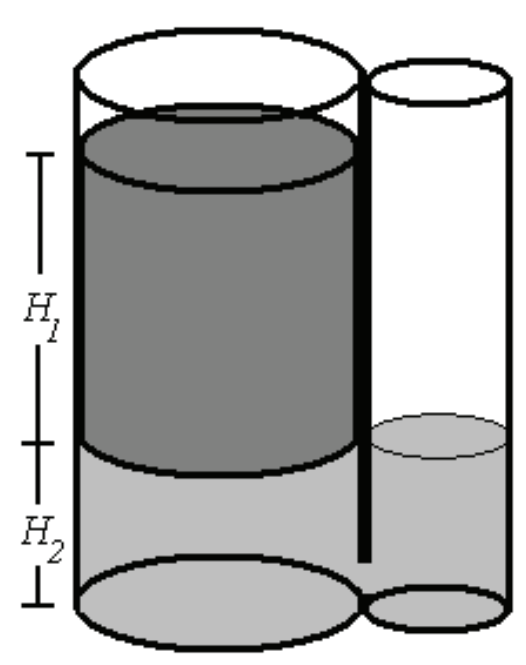

Balança desequilibrada

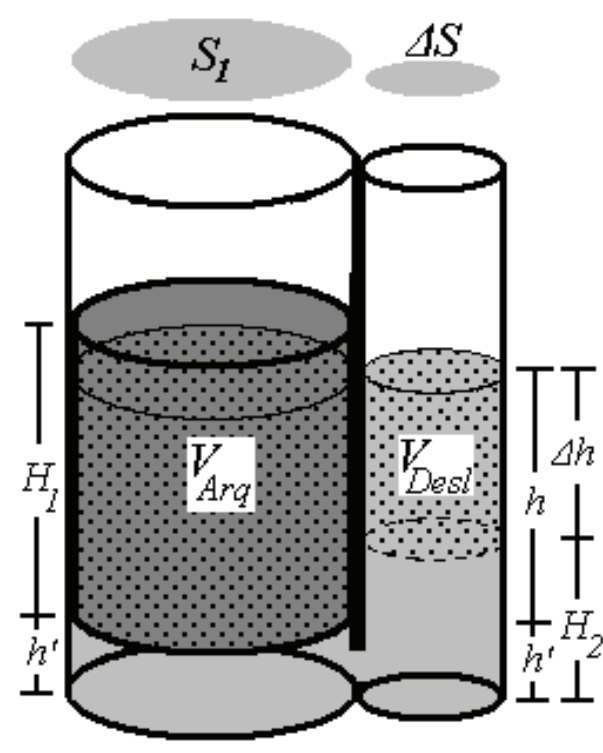

Balança equilibrada

Fig. 5 - Volume $V_{\text {Desl }}$ de líquido deslocado é menor do que o volume $V_{A r q}$ do corpo abaixo do nível da superficie de separação do líquido com a atmosfera. 
O volume de líquido quando a balança está desequilibrada (vide Fig. 5) é dado pela soma dos volumes de líquido nos dois ramos da balança, ou seja

$$
V=S_{1} H_{2}+\Delta S H_{2} \text {. }
$$

Quando a balança está equilibrada, o volume de líquido, que continua o mesmo de antes, pode ser expresso por

$$
V=S_{1} h^{\prime}+\Delta S\left(h^{\prime}+h\right) \text {. }
$$

Igualando entre si as duas expressões obtidas para $V$, em (11) e em (12), e explicitando $h$ ', obtém-se:

$$
h^{\prime}=H_{2}-\frac{\Delta S}{\Delta S+S_{1}} h .
$$

$\mathrm{O}$ volume de líquido deslocado (vide a região assinalada no ramo direito da balança na Fig. 5) é dado por:

$$
V_{\text {Desl }}=\Delta S \Delta h=\Delta S\left(h+h^{\prime}-H_{2}\right) \text {, }
$$

que, após substituir $h$ ' pela expressão obtida em (13), fornece:

$$
V_{\text {Desl }}=\frac{\Delta S}{\Delta S+S_{I}}\left(S_{I} h\right)
$$

O termo entre parênteses em (15) é o volume submerso $V_{A r q}$ do corpo flutuante no líquido. Portanto,

$$
V_{\text {Desl }}=\frac{\Delta S}{\Delta S+S_{l}} V_{\text {arq }}<V_{A r q} .
$$

No caso especial em que $\Delta S>>S_{l}$, pode-se escrever:

$$
V_{\text {Desl }}=\frac{\Delta S}{\Delta S+S_{1}} V_{\text {arq }} \approx V_{A r q},
$$

isto é, $V_{D e s l}$ é um pouco menor que $V_{\text {Arq }}$.

Ou seja, o enunciado usual da Lei de Arquimedes está correto apenas se as dimensões do recipiente que contém o líquido forem muito maiores que as dimensões do corpo ali colocado. Se essa condição estiver satisfeita, o nível do líquido no recipiente permanecerá inalterado quando o corpo for colocado a flutuar; o volume deslocado de líquido será igual ao volume submerso do corpo no líquido. Outra possibilidade de o nível do líquido no recipiente não se alterar ocorre quando o recipiente está repleto de líquido, extravasando fluido devido à imersão do corpo. 


\section{Uma abordagem energética na resolução do Paradoxo de Galileu utilizando o modelo da balança hidrostática}

Apresentaremos agora uma outra forma de resolver o Paradoxo de Galileu, ainda utilizando o modelo da balança hidrostática, mas recorrendo a considerações energéticas.

Partiremos do conhecimento de que qualquer sistema que se encontre em equilíbrio estável satisfaz a condição de que a energia potencial do sistema seja mínima. Dessa forma, se imaginarmos pequenos deslocamentos do sistema em torno de uma posição de equilíbrio estável, deveremos encontrar apenas aumentos na sua energia potencial; caso contrário, a energia potencial não teria sido mínima e o sistema não teria estado anteriormente em equilíbrio.

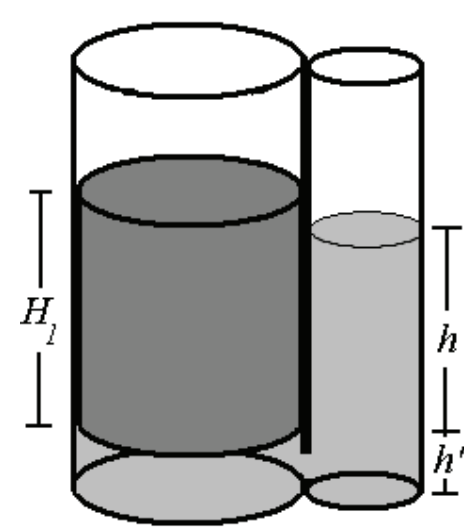

Balança equilibrada

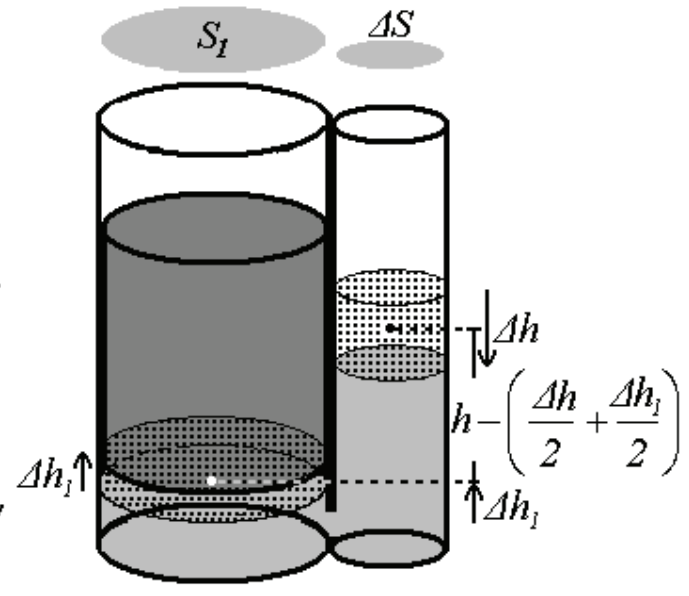

Balança desequilibrada

Fig. 6 - Quando a balança é desequilibrada, sua energia potencial gravitacional deve tornar-se maior que a de equilíbrio.

Vamos agora calcular a variação de energia potencial gravitacional, ao submeter o corpo que flutua a um pequeno deslocamento $\Delta h_{l}$, tal como mostrado na Fig. 6. O líquido no interior do ramo esquerdo se eleva por uma altura $\Delta h_{l}$, enquanto no outro ramo desce por uma altura $\Delta h$, tal que $S_{l} \Delta h_{l}=\Delta S \Delta h$, ou seja,

$$
\Delta h=\frac{S_{1}}{\Delta S} \Delta h_{1} .
$$


A variação $\triangle E p_{\text {Balanca }}$, da energia potencial da balança, é a soma da variação $\Delta E p_{\text {Corpo }}$, da energia potencial do corpo, com a variação $\Delta E p_{\text {Liquido }}$, da energia potencial do líquido, isto é:

$$
\triangle E p_{\text {Balanç }}=\Delta E p_{\text {Corpo }}+\Delta E p_{\text {Liquido }} \text {. }
$$

A variação da energia potencial do corpo é dada por:

$$
\Delta E p_{\text {Corpo }}=m_{\text {Corpo }} g \Delta h_{1},
$$

na qual a massa do corpo pode ser expressa em função da densidade do sólido e do seu volume, como

$$
m_{\text {Corpo }}=\rho_{\text {Sólido }} S_{1} H_{1},
$$

e assim reescrevemos (20) sob a forma:

$$
\Delta E p_{\text {Corpo }}=\rho_{\text {Sólido }} S_{1} H_{1} g \Delta h_{1} .
$$

A variação da energia potencial do líquido é:

$$
\Delta E p_{\text {Liquido }}=m_{\text {Liquido }} g\left(-\Delta h_{\text {Liquido }}\right) \text {, }
$$

podendo a massa de líquido ser expressa em função da densidade do líquido e do volume de líquido, no interior da região assinalada (marcada com pontos escuros) no ramo direito ou no ramo esquerdo da balança, ou seja,

$$
m_{\text {Liquido }}=\rho_{\text {Liquido }} S_{1} \Delta h_{1} \text {, }
$$

e, para a variação de altura do líquido, temos, da Figura 6 e usando (18):

$$
\Delta h_{\text {Liquido }}=h-\left(\frac{\Delta h}{2}+\frac{\Delta h_{1}}{2}\right)=h-\frac{\Delta h_{I}}{2}\left(\frac{S_{I}}{\Delta S}+1\right) .
$$

Assim, reescrevemos (23) sob a forma:

$$
\Delta E p_{\text {Liquido }}=-\rho_{\text {Liquido }} S_{1} \Delta h_{l} g\left(h-\frac{\Delta h_{l}}{2}\left(\frac{S_{I}}{\Delta S}+1\right)\right) \text {. }
$$

Substituindo-se (22) e (26) em (19), e colocando-se alguns fatores em evidência, obtém-se, para a variação da energia potencial da balança, a expressão:

$$
\Delta E p_{\text {Balansa }}=\left[\rho_{\text {Sólido }} H_{1}-\rho_{\text {Liquido }} h\right] S_{1} g \Delta h_{1}+\rho_{\text {Liquido }} S_{1} g\left(\frac{S_{1}}{\Delta S}+1\right) \frac{\Delta h_{1}^{2}}{2} \text {. }
$$

Lembremos que, se a balança estiver na posição de equilíbrio estável (posição de mínima energia potencial), um pequeno afastamento dessa posição em qualquer dos dois sentidos (isto é, $\Delta h_{l}>0$ ou $\Delta h_{1}<0$ ) deverá causar uma variação $\triangle E p_{\text {Balanç }}$ positiva. O segundo termo no lado direito da expressão (27), que envol- 
ve $\Delta h_{l}^{2}$, é sempre positivo. Quanto ao primeiro termo, observamos que, se a diferença entre colchetes resultar não nula, esse termo trocará de sinal quando $\Delta h_{l}$ mudar de orientação. Dessa forma, para garantir que esta variação da energia potencial seja sempre positiva, independentemente da orientação do deslocamento $\Delta h_{l}$, é necessário que:

$$
\left\lfloor\rho_{\text {Sólido }} H_{1}-\rho_{\text {Liquido }} h\right\rfloor=0 \text {, }
$$

isto é,

$$
\rho_{\text {Sólido }}=\frac{h}{H_{1}} \rho_{\text {Liquido }}
$$

O resultado (29) é exatamente o mesmo que obtivemos em (8). Ou seja, chegamos à mesma condição para que ocorra a flutuação; anteriormente o resultado foi derivado diretamente da condição dinâmica de equilíbrio (igualdade entre as pressões em pontos no mesmo nível de um fluido) e agora partindo da condição de que a energia potencial, em um equilíbrio estável, deve ser mínima.

Embora não seja usual em cursos de Física Geral, seja no Ensino Médio ou no Ensino Superior, apresentar uma abordagem do equilíbrio estável, utilizando-se considerações energéticas, não pressupõe qualquer conhecimento de matemática diferente daquele utilizado na seção anterior ou do que utilizaremos na próxima seção.

\section{A Lei de Arquimedes e a Lei de Stevin}

Finalmente, abordaremos o problema do corpo flutuante através da Lei de Stevin ou da Lei Fundamental da Estática dos Fluidos. Para tanto, não faremos nenhuma suposição sobre a geometria do corpo que será colocado a flutuar. A Fig. 7 representa o corpo e o recipiente com líquido, antes e depois de o corpo ser posto a flutuar.

De acordo com a Fig. 7, há uma elevação $\Delta h$ da superfície de separação entre o líquido e a atmosfera, quando o corpo é posto a flutuar. Essa elevação do nível de líquido produz um aumento da pressão sobre o fundo do recipiente que, pela Lei de Stevin, é dado por:

$$
\Delta p=\rho_{\text {Liquido }} g \Delta h,
$$

que, por sua vez, produzirá um acréscimo $\Delta F$, na força que o fluido faz sobre o fundo do recipiente. Representando por $S_{2}$ a superfície do fundo do recipiente, 
podemos escrever o incremento na força exercida pelo fluido no fundo do recipiente sob a forma:

$$
\Delta F=\Delta p S_{2}=\rho_{\text {Liquido }} g \Delta h S_{2} \text {. }
$$
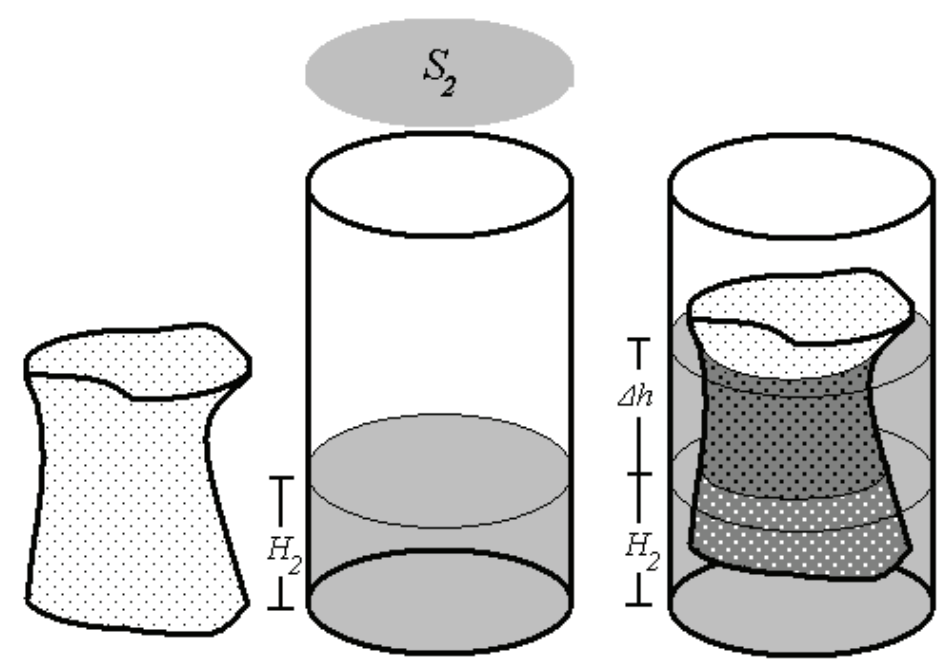

Fig. 7 - O nivel da superficie de separação entre o líquido e a atmosfera sofre uma elevação $\Delta h$, quando o corpo é posto a flutuar, e o volume de líquido deslocado é menor do que o volume expresso no enunciado tradicional da Lei de Arquimedes.

Esse incremento de força sobre o fundo do recipiente também é atribuído ao aumento de peso de todo o material que está contido no recipiente. Ora, o aumento de peso se deve à introdução, no recipiente, do corpo que flutua, cujo peso pode ser escrito em função da densidade do material que o constitui e do seu volume, isto é:

$$
\Delta F=P_{\text {Corpo }}=\rho_{\text {Corpo }} V_{\text {Corpo }} g .
$$

Das expressões (31) e (32) obtém-se:

$$
\rho_{\text {Corpo }} V_{\text {Corpo }} g=\rho_{\text {Liquido }} g \Delta h S_{2}
$$

Na Fig. 8, apresentaremos com detalhe os diversos volumes envolvidos na situação analisada. O produto $\Delta h S_{2}$ é o volume da região assinalada com pontos escuros na Fig. $8 \mathrm{C}$, o qual resulta da soma de duas parcelas, discriminadas na Fig. $8 \mathrm{D}$, a saber: um volume $V^{\prime}$, da parte do corpo que flutua, situada dentro do líquido 
mas acima do nível original $H_{2}$ da superfície de contato entre o líquido e a atmosfera, e um volume $V_{\text {Desl }}$ do líquido que foi deslocado, isto é:

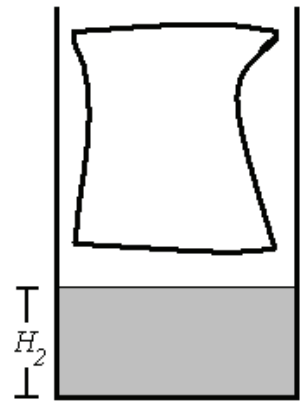

Figura $\mathrm{A}$

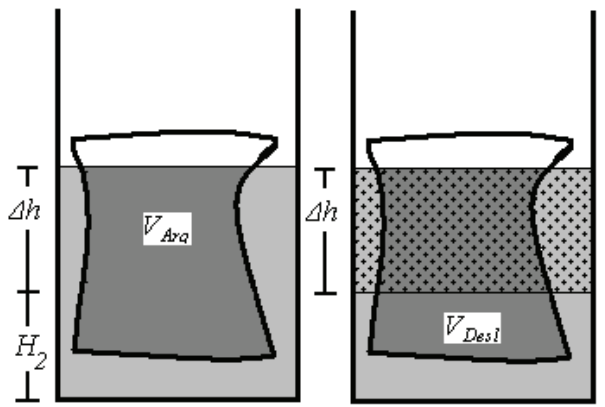

Figura B

Figura $\mathrm{C}$

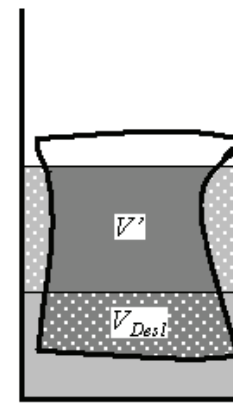

Figura D

Fig. 8 - O nível do líquido se eleva por uma altura $\Delta h$, após introduzir no líquido o corpo flutuante.

$$
\Delta h S_{2}=V^{+}+V_{\text {Desl }},
$$

no qual $V_{\text {Desl }}$ é o volume de líquido que foi efetivamente deslocado, elevando-se acima do nível original $H_{2}$, da superfície de contato entre o líquido e a atmosfera da Fig. 8 A.

Por outro lado, observe-se, das regiões assinaladas com pontos brancos na Fig. $8 \mathrm{D}$, que o volume de líquido que foi deslocado, elevando-se acima do nível original do líquido, é igual ao volume da parte do corpo situada abaixo deste nível; ambos são iguais a $V_{\text {Desl }}$. Concluímos, portanto, que a soma $V^{\prime}+V_{\text {Desl }}$ é igual ao volume $V_{A r q}$, da parte do corpo situada abaixo do novo nível da superfície do líquido (vide Fig. 8 B). Ou seja,

$$
V^{\prime}+V_{\text {Desl }}=V_{\text {Arq }} \text {. }
$$

De imediato, observamos que da igualdade (35) decorre que o volume $V_{A r q}$ é maior do que o volume de líquido deslocado $V_{\text {Desl }}$. Somente quando o nível do líquido não se eleva pela introdução do corpo flutuante (isso ocorre quando as dimensões do corpo são desprezíveis em relação às dimensões do recipiente ou quando o recipiente, inicialmente repleto de líquido, transborda quando o corpo é posto a flutuar) é que $V^{\prime}=0$ e então $V_{D e s l}=V_{A r q}$. Em qualquer outra situação $V_{D e s l}$ $<V_{\text {Arq }}$.

De (35) juntamente com (34), obtém-se:

$$
\Delta h S_{2}=V_{A r q} \text {, }
$$


que, substituído na expressão (33), fornece:

$$
\rho_{\text {Corpo }} V_{\text {Corpo }} g=\rho_{\text {Liquido }} V_{\text {Arq }} g \text {. }
$$

A condição de flutuação é finalmente assim expressa: o peso do corpo deve ser igual ao peso do líquido contido em um volume idêntico ao volume submerso do corpo no líquido $\left(V_{A r q}\right)$.

A igualdade (37) pode ser reescrita sob a forma:

$$
\frac{\rho_{\text {Corpo }}}{\rho_{\text {Liquido }}}=\frac{V_{\text {Arq }}}{V_{\text {Corpo }}},
$$

donde conclui-se imediatamente que:

$$
\frac{\rho_{\text {Corpo }}}{\rho_{\text {Liquido }}} \leq 1 \Rightarrow \rho_{\text {Corpo }} \leq \rho_{\text {Liquido }},
$$

pois, para um corpo flutuar, sempre tem-se $V_{\text {Corpo }} \geq V_{A r q}$.

Assim, fica demonstrada a conhecida condição (39) de flutuação, que afirma que o corpo flutuará se a densidade do material que o constitui (ou a densidade média do corpo) for no máximo igual à do líquido. Essa condição já tinha sido obtida nas seções anteriores, onde supusemos um corpo cilíndrico e partimos do modelo da balança hidrostática; agora a obtivemos sem fazer qualquer suposição sobre a forma do corpo que flutua.

\section{Conclusão}

É inquestionável que o enunciado tradicional da Lei de Arquimedes está equivocado. Lembremos de novo que a formulação usual da Lei de Arquimedes é a seguinte: todo corpo mergulhado em um líquido sofre um empuxo de baixo para cima igual ao peso do fluido por ele deslocado.

Evidencia-se um erro na formulação usual da Lei de Arquimedes no Paradoxo Hidrostático de Galileu, pois um corpo pode flutuar em um fluido mesmo quando o peso de todo o fluido disponível é menor do que o peso do corpo.

Para que a Lei de Arquimedes evite, não propicie o Paradoxo Hidrostático de Galileu, deve ser formulada do seguinte modo: todo corpo mergulhado em um líquido sofre um empuxo de baixo para cima igual ao peso do fluido contido em um volume idêntico ao volume submerso do corpo no fluido.

Da análise realizada neste artigo, depreende-se a extensão em que o ignorar de certos pressupostos presentes na formulação de determinadas leis na Física 
pode se constituir em sérios obstáculos epistemológicos para a compreensão do contexto de aplicação e/ou de validade de tais leis. O caso aqui especificamente tratado, o enunciado usual da Lei de Arquimedes, que dá origem ao Paradoxo Hidrostático de Galileu, surge efetivamente do fato de se subestimar as implicações das dimensões do recipiente que contém o fluido e o corpo imerso. As abordagens das condições de flutuação aqui apresentadas tentam romper esse discurso ideológico tradicional, repleto de lacunas, calcado em pressupostos empiristasindutivistas, que pretende que de experimentos se possa induzir as leis da natureza, tão presente na pedagogia tradicional do ensino da Física. Este, certamente, não é um caso isolado no ensino da Física, mas se oferece como um exemplo, dentre outros possíveis, para demonstrar que análises desse tipo podem contribuir positivamente, não apenas para melhorar a formação dos nossos colegas professores de Física do Ensino Médio, mas também para auxiliar no desvendar de certas dúvidas que os estudantes costumeiramente apresentam em sua aprendizagem.

\section{Agradecimentos}

Agradecemos à Prof ${ }^{\mathrm{a}}$ Maria Cristina Varriale, do IM-UFRGS, e à Prof ${ }^{\mathrm{a}}$ Eliane Veit, do IF-UFRGS, pela leitura crítica deste artigo e pelas valiosas sugestões apresentadas.

\section{Referências Bibliográficas}

ASSIS, A. K.T. Sobre os corpos flutuantes - tradução comentada de um texto de Arquimedes. Revista da Sociedade Brasileira de História da Ciência, v. 16, p. 69-80, 1996.

BARDIS, P. An Ancient Find. Science Teacher, v. 51, n. 7, p. 32-33, October 1984.

BARTLETT, A. The Hydrostatic Paradox revisited. The Physics Teacher, v. 35, n. 5, p. 288-289, May 1997.

BETYAEV, S. Hydroparadoxes: when fluids forsake model behavior. Quantum, p. 20, July-August 1998.

BUZDIN, A.; KROTOV, S. Boy-Oh-Buoyancy! Problems in Fluid Statics. Quantum, p.27, September-October 1990. 
COOPER, S. In The footsteps of Archimedes. Science Teacher, v. 68, n. 1, p. 3435, January 2001.

DUGAS, R. A History of Mechanics. New York: Dover Publications, 1988.

FIOLHAIS, C. Física Divertida. Lisboa: Gradiva, 1999.

FONTANA, F.; DI CÁPUA, R. Role of hydrostatic paradoxes towards the formation of the scientific thought of students at academic level. European Journal of Physics, v. 26, n. 6, p. 1017-1030, November 2005.

GUIMARÃES, A. O velho Princípio de Arquimedes. Caderno Catarinense de Ensino de Física, v. 16, n. 2, 1999.

HALFORD, G.; BROWN, C.; THOMPSON, R. Children's Concepts of Volume and Flotation. Developmental Psychology, v. 22, p. 218-222, 1986.

HAYN, C. Archimedes and the Magdeburg Hemispheres. The Physics Teacher, v. 13, n. 1, p. 42-43, January 1975.

HODDESON, L. How did Archimedes solve king hiero's crown problem? An unanswered question. The Physics Teacher, v. 10, n. 1, p. 14-19, January 1972.

HUGHES, S. Archimedes revisited: a faster, better, cheaper method of accurately measuring the volume of small objects. Physics Education, v. 40, n. 5, p. 468474, September 2005.

KAPLAN, G. Eureka! Or don't throw out the crown with the bathwater. Mathematics Teaching in the Middle School, v. 8, n. 9, p. 484-488, May 2003.

LALANDE, A. Vocabulário técnico e crítico da Filosofia. São Paulo: Martins Fontes, 1993.

LOVERUDE, M.; KAUTZ, C.; HERON, P. Helping students develop an understanding of Archimedes' Principle. I. Research on Student Understanding. American Journal of Physics, v. 71, n. 11, p. 1178-1187, November 2003.

MACH, E. The Science of Mechanics. La Salle, Illinois: Open Court Classics, 1989. 
MAMOLA, K. Archimedes' Principle and smiley-faced balloons. The Physics Teacher, v. 33, n. 3, p. 172-173, March 1995.

MARTINS, R. Arquimedes e a coroa do rei: problemas históricos. Caderno Catarinense de Ensino de Física, v. 17, n. 2, p. 115-121, 2000.

MEDEIROS, A.; MEDEIROS, C. Desvendando o mistério do duplo cone. Revista Brasileira de Ensino de Física, v. 25, n. 3, Setembro 2003.

MEDEIROS, A. A Física com o Circo e o Circo sem a Física. Folhetim da Física, Junho de 2005. Disponível em:

$<$ http://www.galeradafisica.com.br/fe/23/vanDeGraaff.pdf $>$

MEDEIROS, C.; MEDEIROS, A. Brincando e Aprendendo com o Mistério. ABCeducatio, v. 5, n. 37, Agosto 2004.

MEDEIROS, A.; MEDEIROS, C. Considerações sobre os contextos da descoberta e da justificativa na Álgebra de Pedro Nunes. Episteme, v. 15, Agosto-Dezembro 2002.

MEDEIROS, A.; CARMO, L. Problematizando o uso de Mergulhadores de Descartes no ensino da Hidrostática em um Curso de Engenharia de Pesca. Revista de Engenharia, Novembro 2003.

MEDEIROS, A; MEDEIROS, C.; CARMO, L. Modelagens e subsunçores na compreensão do funcionamento de um Mergulhador de Descartes. In: ENCONTRO INTERNACIONAL DE APRENDIZAGEM SIGNIFICATIVA, IV, 2003, Maragogi, Alagoas. Atas...

MEDEIROS, A. A prensa hidráulica na sala de aula: de onde vem os nove? Folhetim da Física, Novembro 1999.

MEDEIROS, A.; MEDEIROS, C. F. Questões epistemológicas nas iconicidades de representações visuais em livros didáticos de Física. Revista Brasileira de Pesquisas em Educação em Ciências, v. 1, n. 1, 2001.

MEDEIROS, A.; LIMA Jr., N. Identificando pressupostos e contextos de validade em experimentos com eletroscópios. In: ENCONTRO DE PESQUISADORES EM ENSINO DE FÍSICA, VII, 2000, Florianópolis. 
MEDEIROS, A.; BEZERRA FILHO, S. A natureza da ciência e a instrumentação para o ensino da Física. Educação \& Ciência, v. 6, n. 2, p. 107-117, 2000.

MOURA, R.; CANALLE, J. Os mitos dos cientistas e suas controvérsias. Revista Brasileira de Ensino de Física, v. 23, n. 2, Junho 2001.

SCONYERS, J.; TRAUTWEIN, C. Putting a spring in Archimedes' step. Science Scope, v. 23, n. 5, p. 14-16, February 2000.

SILVA, A. Archimedes' Law and potential energy: modelling and simulation with a spreadsheet. Physics Education, v. 33, n. 2, p. 87-92, March 1998.

SILVEIRA, F. L.; AXT, R. O que vemos quando projetamos a luz do Sol com um espelho plano: manchas luminosas ou imagens? Caderno Brasileiro de Ensino de Física, v. 24, n. Especial, p. 246-257, 2004.

SILVEIRA, F. L.; AXT, R. Podem molas em queda livre ter aceleração maior do que a da gravidade? A Física na Escola, v. 6, n. 2, p. 5-7, 2005.

Uma dificuldade recorrente em Óptica Geométrica - Uma imperceptível descontinuidade de imagem na lupa. Revista Brasileira de Ensino de Física, v. 28, n. 4, p. 421-425, 2006.

SILVEIRA, F. L.; LEVIN, Y. Pressão e volume em balões de festa: podemos confiar em nossa intuição? Caderno Brasileiro de Ensino de Física, v. 21, n. 3, p. 285-295, 2004.

SILVEIRA, F. L.; MEDEIROS, A. A ilusão do tamanho da lua no horizonte. A Física na Escola, v. 7, n. 2, p. 67-69, 2006.

SILVEIRA, F. L.; OSTERMANN, F. A insustentabilidade da proposta indutivista de descobrir a lei a partir de resultados experimentais. Caderno Brasileiro de Ensino de Física, v. 19, p. 7-27, 2002.

SILVEIRA, F. L.; PEDDUZI, L. O. Q. Três episódios de descoberta científica: da caricatura empirista a uma outra História. Caderno Brasileiro de Ensino de Física, v. 23, n. 1, p. 26-52, 2006.

SNIR, J. Sink or float - What do experts think?: The historical development of explanations for floatation. Science Education, v. 75, n. 5, p. 595-609, 1991. 
TOKATY, G. A History and Philosophy of Fluid Mechanics. New York: Dover Publications, 1994.

WILSON, A. The Hydrostatic Paradox. The Physics Teacher. v. 33, n. 8, p. 538539, November 1995. 